\section{ralud mental}

Barrera Villalpando, María Isabel; Cortés Sotres, José Francisco; Mendieta Cabrera,

Danelia; Guerrero León, Daniela

Disminución del influjo parasimpático en fibromialgia: Su relación con la Psiquiatría en un centro especializado de referencia nacional

Salud Mental, vol. 38, núm. 2, marzo-abril, 2015, pp. 123-128

Instituto Nacional de Psiquiatría Ramón de la Fuente Muñiz

Distrito Federal, México

Disponible en: http://www.redalyc.org/articulo.oa?id=58239238007

- Cómo citar el artículo

Número completo

- Más información del artículo

Página de la revista en redalyc.org

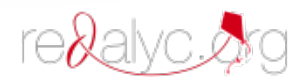

Sistema de Información Científica

Red de Revistas Científicas de América Latina, el Caribe, España y Portugal Proyecto académico sin fines de lucro, desarrollado bajo la iniciativa de acceso abierto 


\title{
Disminución del influjo parasimpático en fibromialgia: Su relación con la Psiquiatría en un centro especializado de referencia nacional
}

\author{
María Isabel Barrera Villalpando, ' José Francisco Cortés Sotres, ${ }^{2}$ Danelia Mendieta Cabrera, \\ Daniela Guerrero León ${ }^{3}$
}

Artículo original

\section{ABSTRACT}

\section{Background}

Fibromyalgia (FM) is characterized by chronic widespread pain, fatigue, sleep disturbances, depression, anxiety and dysautonomia (sympathetic hyperactivity).

\section{Objective}

To compare the heart rate variability (HRV) in women: 20 patients with FM vs. 20 controls by Holter 24 hrs.

\section{Method}

The measurement consisted of segments of five minutes. The frequency domain is determined by the natural logarithm of the LF/HF (Low/ High Frecuencies) reason. Simple ANOVA was used for two groups of dimensional variables.

\section{Results}

The age range was $30-60$ years. Nine presented psychiatric comorbidity: depression $(77.7 \%)$ and anxiety $(22.3 \%)$. There were differences $(\mathrm{F}=24.45, \mathrm{p}<0.0001)$ in $\mathrm{LF} / \mathrm{HF}$ between groups in the nocturnal phase of registration (22 pm to $2 \mathrm{am}$ ) showing increased sympathetic activation in patients. In the SDNN index (standard deviation of intervals between heartbeats) there were significant differences on December 9 periods of record. In pNN50 index (percentage of intervals which differ by more than 50 milliseconds), the control group showed higher values of 6 to $12 \mathrm{hrs}$. Nocturnal variation was observed in 22 hrs $(F=22.37, p=0.0001)$ until $6 a m(F=30.27, p=0.0001)$. The rMSSD indicator (square root of the mean of the differences in heart rate) showed higher values for the control group from $22 \mathrm{hrs}$ (F = $67.71, p=0.0001)$ until 6am ( $F=80.35, p=0.0001)$.

\section{Discussion and conclusion}

The results reflect the decreased parasympathetic influence in patients with FM.

This confirms the participation of parasympathetic nervous system in the pathophysiology of FM.

Key words: Fibromyalgia, chronic pain, disautonomy, heart rate variability, depression, anxiety.

\section{RESUMEN}

\section{Antecedentes}

La fibromialgia (FM) se caracteriza por dolor crónico generalizado, fatiga, alteraciones del sueño, depresión, ansiedad y disautonomía (hiperactividad simpática).

\section{Objetivo}

Comparar la variabilidad de la frecuencia cardiaca (VFC) en mujeres: 20 pacientes con FM vs. 20 controles, mediante Holter de 24 hrs.

\section{Método}

La medición consistió en segmentos de cinco minutos. El dominio de la frecuencia se determinó por logaritmo natural de la razón LF/HF (Low/High Frecuencies). Se utilizó ANOVA simple para dos grupos de variables dimensionales.

\section{Resultados}

El rango de edad fue de 30 a 60 años. Nueve mujeres presentaron comorbilidad psiquiátrica: depresión (77.7\%) y ansiedad (22.3\%). Hubo diferencias ( $F=24.45, p<0.0001$ ) en $L F / H F$ entre los grupos en la fase nocturna del registro (22 hrs a 2 am), mostrándose mayor activación simpática en las pacientes. En el índice SDNN (desviación estándar de intervalos entre latidos) existieron diferencias significativas en 9 de 12 periodos del registro. En el índice pNN50 (porcentaje de intervalos que difieren en más de 50 milisegundos), el grupo control mostró valores más altos de 6 a 12 hrs. La variación nocturna se observó de 22 hrs. ( $F=22.37, p=0.0001)$ hasta las 6 am $(F=30.27, p=0.0001)$. El indicador rMSSD (raíz cuadrada de la media de las diferencias de la frecuencia cardiaca) mostró valores más altos para el grupo control desde las 22 hrs. ( $F=67.71, p=0.0001)$ hasta las $6 a m(F=80.35, p=0.0001)$.

\section{Discusión y conclusión}

Los resultados reflejan la disminución del influjo parasimpático en las pacientes con FM.

Esto confirma la participación del sistema nervioso parasimpático en la fisiopatología de la FM.

Palabras clave: Fibromialgia, dolor crónico, disautonomía, variabilidad de la frecuencia cardiaca, depresión y ansiedad.

\section{Dirección de Servicios Clínicos. Instituto Nacional de Psiquiatría Ramón de la Fuente Muñiz. \\ 2 Dirección de Enseñanza. Instituto Nacional de Psiquiatría Ramón de la Fuente Muñiz. \\ Facultad de Medicina. Universidad Nacional Autónoma de México.}

Correspondencia: Dra. María Isabel Barrera Villalpando. Dirección de Servicios Clínicos. Instituto Nacional de Psiquiatría Ramón de la Fuente Muñiz. Calz. México-Xochimilco 101, San Lorenzo Huipulco, Tlalpan, 14370, México, DF. E-mail: barma@imp.edu.mx

Recibido primera versión: 20 de abril de 2013. Segunda versión: 11 de abril de 2014. Aceptado: 15 de julio de 2014. 


\section{ANTECEDENTES}

La fibromialgia (FM) se conoce como un síndrome crónico caracterizado fundamentalmente por la presencia de dolor generalizado, difuso y crónico, sin anomalías aparentes, y se acompaña de otros síntomas tales como fatiga, alteración del sueño, parestesias, problemas de concentración, ansiedad y depresión ${ }^{1,2}$ generando importantes cambios en el funcionamiento físico, psicológico y social de quienes lo padecen. Diversos estudios muestran que la población afectada es del dos al 5\%, ${ }^{3}$ siendo más frecuente en las mujeres, con una media de edad inicial que varía entre los 30 y los 60 años. ${ }^{4}$ Aunque no se cuenta aún con una clara etiología, las investigaciones actuales señalan una disfunción neuroendocrinoinmunológica, ${ }^{5}$ problemas de sensibilización central ${ }^{6}$ así como una alteración en el Sistema Nervioso Autónomo (SNA). Este último, es altamente adaptable y permite al organismo mantener su equilibrio cuando se experimenta estrés. La falta de flexibilidad y un sistema rígido puede conducir a patologías somáticas y psicológicas. ${ }^{7}$ Una de las formas como se mide esta respuesta es por medio de la variabilidad de la frecuencia cardiaca (VFC); ésta puede estudiarse en su dimensión tiempo (milisegundos), sea por medio de la desviación estándar (SDNN) de todas las poblaciones de los intervalos entre latidos, o por medio del porcentaje de pares adyacentes de estos intervalos que difieren en más de 50 milisegundos uno del otro (pNN50) durante un periodo de tiempo determinado. Un mayor índice de variabilidad en la dimensión temporal significa una mayor influencia parasimpática. Otro índice para evaluar la variabilidad de la frecuencia cardiaca es la raíz cuadrada de la media sucesiva de las diferencias de la frecuencia cardiaca (rMSSD). Su medición respecto a la dimensión frecuencia, se realiza mediante un análisis espectral y brinda información sobre la varianza (poder) en el ritmo cardiaco explicado a través de oscilaciones periódicas de la frecuencia cardiaca (FC) en diferentes bandas de frecuencia (BF) ${ }^{8,9}$ La banda de alta frecuencia (HF) representa la acción del Sistema Nervioso Parasimpático (SNP), mientras que la banda de baja frecuencia $(\mathrm{LH})$ refleja un mayor predominio de la actividad simpática. ${ }^{8,10}$ Un estudio ${ }^{11}$ reveló que ante un estresor ortostático, al comparar a los pacientes con FM con controles, los primeros no pudieron aumentar la BF en respuesta a ponerse de pie. En otro estudio, ${ }^{12}$ se evaluó el comportamiento circadiano del SNA mediante un Holter durante 24 horas mientras desarrollaban sus actividades diarias y tanto el análisis en la dimensión tiempo como en el de la frecuencia, demostraron que las pacientes con FM tenían alteraciones compatibles con una hiperactividad simpática incesante durante el día, pero particularmente evidente durante la noche. De allí que varios estudios reporten que las pacientes con FM presentan una disautonomía consistente en una hiperactividad del Sistema Nervioso Simpático (SNS) que se torna hiporreactivo al estrés. ${ }^{13-15}$ Sin embargo, las alteraciones de la VFC también se ha documentado en otros padeci- mientos psiquiátricos. En comparación con controles sanos y controles no psiquiátricos se ha encontrado un índice LH/HF mayor en los pacientes con trastorno depresivo mayor (TDM) y trastornos de ansiedad ${ }^{16,17}$ lo que indica una disminución del tono vagal, reflejando un déficit en la flexibilidad de los mecanismos fisiológicos. ${ }^{7}$ Algunos estudios ${ }^{18}$ señalan la asociación entre la depresión, la reducción de VFC y el uso de antidepresivos, ${ }^{19}$ enfatizando la relación entre depresión mayor y trastornos cardiovasculares, mediado esto, en parte, por la reducción de la VFC. ${ }^{20}$ La alta prevalencia reportada entre FM y TDM, ${ }^{21-26}$ junto con los hallazgos de una VFC disminuida en pacientes con alguna condición de dolor crónico, ${ }^{27}$ obliga a tomar en cuenta tratamientos como la terapia cognitivo conductual (TCC), la estimulación magnética transcraneal (EMT) y la retroalimentación biológica (RB)para aumentar la $\mathrm{VFC}^{28}$ y ayudar a controlar los síntomas depresivos..$^{29,30}$ Un punto de partida importante es identificar las diferencias de la VFC en controles y en pacientes con FM que además presentan trastornos psiquiátricos como pretende este estudio.

\section{MÉTODO Diseño}

Es un estudio comparativo y transversal con dos muestras independientes.

\section{Participantes}

La muestra se obtuvo con pacientes del Instituto Nacional de Psiquiatría Ramón de la Fuente Muñiz que asistían a la terapia grupal cognitivo conductual para pacientes con fibromialgia. Los criterios de inclusión fueron: mujeres, de edad entre 30 y 60 años con el diagnóstico de FM y como criterios de exclusión: comorbilidad con otra condición reumatológica o cardiovascular, estados psicóticos o embarazo. Así, la muestra se conformó de cuarenta mujeres en dos grupos: 20 pacientes con FM y 20 controles apareadas por edad ( \pm 2 años).

Las controles fueron profesionales de la salud (psicólogas, médicos, trabajadoras sociales, nutriólogas y enfermeras) y personal administrativo del Instituto Nacional de Psiquiatría. Cabe mencionar que ninguna de ellas, principalmente enfermeras, laboraba en jornada nocturna. Durante el registro, pacientes y controles evitaron el consumo de refrescos de cola, así como de tabaco y cafeína. Todas las participantes firmaron su consentimiento informado.

\section{Procedimiento}

Las participantes fueron evaluadas con la Entrevista Internacional Neuropsiquiátrica (M.I.N.I.) ${ }^{31}$-basada en el DSM-IV y en el CIE-10 en su versión en español y computarizada- la cual fue aplicada por un psiquiatra para determinar si pre- 
Cuadro 1. Datos demográficos de pacientes con fibromialgia y controles

\begin{tabular}{lccccc}
\hline & \multicolumn{2}{c}{ Fibromialgia } & & \multicolumn{2}{c}{ Control } \\
\cline { 2 - 3 } \cline { 6 - 7 } & Media & D. Std. & & Media & D. Std. \\
\hline Edad [años] & $\mathrm{N}$ & $\%$ & & $\mathrm{~N}$ & $\%$ \\
\hline Escolaridad [años] & 14.30 & 4.10 & & 15.90 & 3.20 \\
\hline \multicolumn{1}{c}{} & & & & & \\
\hline Estado civil & 7 & 35 & & 9 & 45 \\
Sin pareja & 13 & 65 & & 11 & 55 \\
$\begin{array}{l}\text { Con pareja } \\
\text { Ocupación }\end{array}$ & & & & \\
Trabajo & 12 & 60 & & 19 & 95 \\
remunerado & 8 & 40 & & 1 & 5 \\
Trabajo no remunerado & & & & & \\
\hline
\end{tabular}

sentaban algún diagnóstico psiquiátrico. En un consultorio de la Dirección de Servicios Clínicos, se citó dos veces a cada una de las participantes, la primera para la explicación del procedimiento, firma de su consentimiento y colocación de una grabadora Holter (monitor ambulatorio para el registro electrocardiográfico de 24 horas), modelo DMS 300-7. En la segunda se retiró la grabadora y se descargaron los datos en un software para su posterior revisión y análisis. El registro inició entre las 8 y las 8.30 hrs.

\section{Análisis estadístico}

Las variables categóricas se presentan en frecuencia y porcentajes, las dimensionales con medias y desviaciones estándar. La unidad de análisis constó de segmentos de cinco minutos. Para el análisis del dominio de la frecuencia se determinó el logaritmo natural de la razón LF/HF. Se analizó la esfericidad de los datos encontrando que su matriz de covarianza difería de la matriz identidad por lo que los datos se consideraron independientes a lo largo del tiempo. Se utilizó ANOVA simple para dos grupos y para las variables dimensionales en estudio. Para el análisis se utilizó el paquete estadístico SPSS versión 18.

\section{RESULTADOS}

La media de edad para el grupo con FM fue de 49.8 años y para el grupo control fue de 47.5 años. En cuanto a su escolaridad, las pacientes tenían en promedio 14.3 años de es-

Cuadro 2. Diagnósticos de las pacientes con fibromialgia

\begin{tabular}{lcccccc}
\hline & \multicolumn{3}{c}{ Primer $\mathrm{Dx}$} & & \multicolumn{2}{c}{ Segundo Dx } \\
\cline { 2 - 3 } Diagnóstico psiquiátrico & $\mathrm{N}$ & $\%$ & & $\mathrm{~N}$ & $\%$ \\
\hline Distimia & 0 & 0 & & 1 & 11.1 \\
Trastorno de ansiedad generalizada & 2 & 22.3 & & 2 & 22.3 \\
Trastorno de angustia & 0 & 0 & & 3 & 44.3 \\
Trastorno depresivo mayor & 7 & 77.7 & 2 & 22.3 \\
\hline
\end{tabular}

Cuadro 3. Tratamiento y comorbilidad psiquiátrica de las pacientes con fibromialgia

\begin{tabular}{lll}
\hline Tratamiento psiquiátrico & $\mathrm{N}$ & $\%$ \\
\hline Antidepresivo dual & 2 & 20 \\
Antidepresivo ISRS & 5 & 50 \\
Antidepresivo tricíclico & 2 & 20 \\
Modulador del dolor & 3 & 30 \\
Benzodiacepina & 1 & 10 \\
\hline
\end{tabular}

tudio, mientras que el grupo control contaba con 15.9 años. De las pacientes, 13 tenían pareja y siete no contaban con ella, en tanto que en el grupo control 11 sí la tenían y nueve no. El grupo control estuvo conformado básicamente por empleadas (19) mientras que sólo 12 de éstas tenían trabajo remunerado (cuadro 1).

Las pacientes fueron diagnosticadas según los criterios del Colegio Americano de Reumatología ${ }^{32}$ con severidad de moderada a severa y con evolución media de siete años. Nueve pacientes presentaron diagnóstico psiquiátrico. Como primer diagnóstico se encontró al trastorno depresivo mayor (TDM) en un $77.7 \%$, seguido del trastorno por ansiedad generalizada (TAG) con un $22.3 \%$. Ocho de las nueve pacientes presentaron más de un diagnóstico psiquiátrico. La mayor comorbilidad fue con el trastorno de angustia $(44.3 \%)$ y con TAG y distimia (22.3\%) (cuadro 2 ).

El tratamiento psiquiátrico empleado fue con inhibidores selectivos de la recaptura de serotonina (ISRS) en un $50 \%$, con antidepresivos tricíclicos en un $20 \%$, antidepresivos duales en otro $20 \%$ y el $10 \%$ restante corresponde a otros antidepresivos. El 30\% de las pacientes tomaba modulador del dolor y sólo una paciente usaba benzodiacepinas (cuadro 3).

En cuanto al índice LF/HF, se encontró una diferencia altamente significativa entre ambos grupos $(\mathrm{F}=24.45$, $\mathrm{p}<0.001$ ) para el período de las 22 a las $24 \mathrm{hrs}$, siendo la media más alta para el grupo de las pacientes, lo que refleja mayor activación simpática. De las 0 a las 2am también se encontró diferencia significativa $(F=5.01, p<0.05)$ entre los grupos, con mayor activación para las pacientes. Entre las 4 y 6am se observó una tendencia a la significancia $(F=3.17$, $\mathrm{p}=0.0754$ ) que también indica mayor actividad simpática en las pacientes. En el periodo comprendido entre las 8 y 10 de la mañana hubo una tendencia a la significancia $(F=3.36$, $\mathrm{p}=0.0670$ ) lo que indica una activación ligeramente mayor para el grupo control (figura 1).

En el índice SDNN se encontraron diferencias significativas entre los grupos básicamente para la mayoría de los períodos(en nueve de un total de 12) que comprendieron las 24 hrs de medición. Sí hubo diferencia entre los grupos para el período entre las 8 y 10am así como para el período entre las 20 y 22 hrs (figura 2).

En el índice pNN50 se encontró que los grupos difirieron en varios periodos de la medición, destacándose los intervalos matutinos de las 6 a 8 hrs $(\mathrm{F}=52.44, \mathrm{p}=0.001)$, de las 8 a 10 hrs $(\mathrm{F}=15.89, \mathrm{p}=0.001)$ y de las 10 a 12 hrs $(\mathrm{F}=10.64$, 


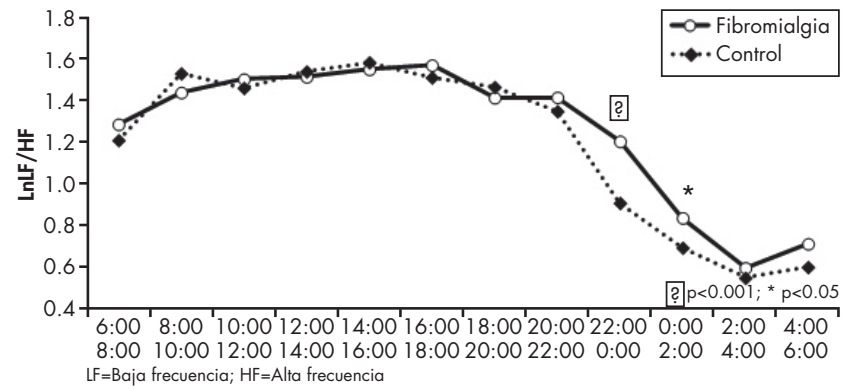

Figura 1. Diferencias en el índice LF/HF entre pacientes con fibromialgia y controles.

$\mathrm{p}=0.001)$. De las 12 a 14 hrs se encontró diferencia estadísticamente significativa $(\mathrm{F}=5.60, \mathrm{p}=0.0183)$. No se observó diferencia entre los grupos de las 14 a 16 hrs. Por la tarde, de las 16 a 18 hrs, se encontró diferencia significativa $(\mathrm{F}=31.6, \mathrm{p}=0.001)$ y la variación nocturna se observó desde las 22 hrs $(\mathrm{F}=22.37, \mathrm{p}=0.001)$ abarcando también de las 0 a 2 a.m. $(\mathrm{F}=40.91, \mathrm{p}=0.001)$. En el período entre las 2 y $4 \mathrm{am}$ se encontró diferencia estadísticamente significativa $(\mathrm{F}=5.24$, $\mathrm{p}=0.0224)$ así como en el período de las 4 a 6 a.m. ( $\mathrm{F}=30.27$, $\mathrm{p}=0.001$ ) (figura 3).

Finalmente, para el indicador rMSSD se obtuvieron valores más altos de las medias (en casi la totalidad de los períodos) para el grupo control sobre todo hacia la noche presentando diferencias significativas de las 20 a las 22 hrs $(\mathrm{F}=11.72, \mathrm{p}=0.006)$; de las 22 a las $24 \mathrm{hrs}(\mathrm{F}=67.71$, $\mathrm{p}=0.001)$; de las 0 a 2 a.m. $(\mathrm{F}=87.16, \mathrm{p}=0.001)$; de las 2 a 4 a.m. $(\mathrm{F}=38.35, \mathrm{p}=0.001)$ y de las 4 a 6 a.m. $(\mathrm{F}=80.35, \mathrm{p}=0.001)$. No se encontraron diferencias en el periodo comprendido entre las 18 a 20 hrs (figura 4).

Se corrobora que el índice pNN50 está altamente correlacionado con el rMSSD, r=0.974 para FM y r=0.986 para el grupo control.

\section{DISCUSIÓN Y CONCLUSIÓN}

Los resultados de nuestro estudio confirman el fenómeno de la disautonomía en pacientes con fibromialgia. Se observan

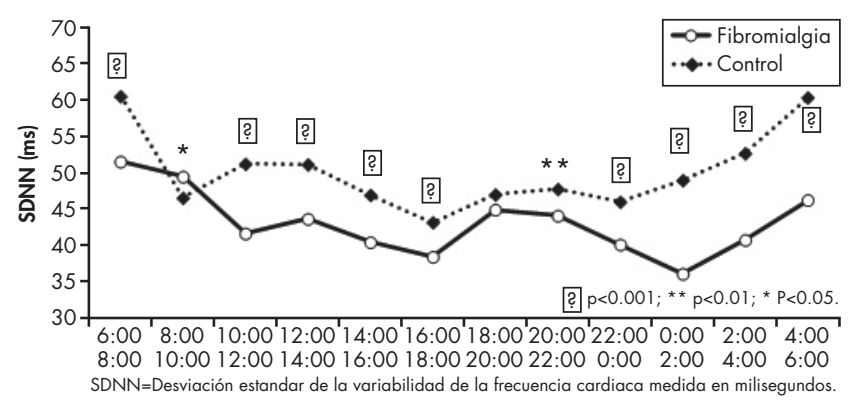

Figura 2. Diferencias en el índice SDNN entre pacientes con fibromialgia y controles.

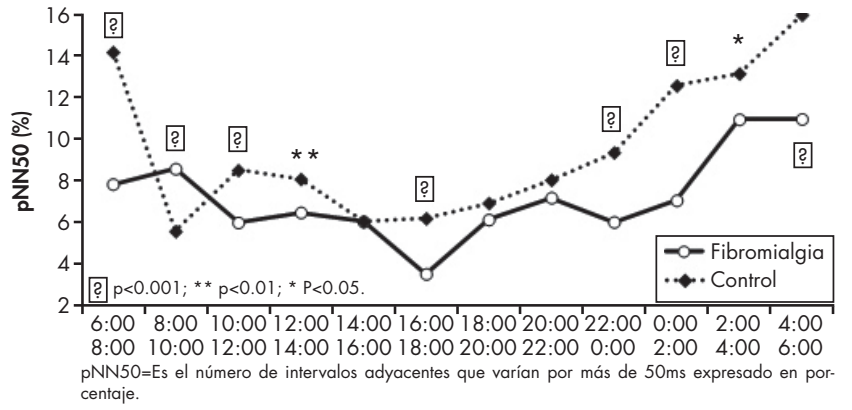

Figura 3. El 70 índice pNN50 en pacientes con fibromialgia y controles.

claras diferencias en el índice LF/HF entre las pacientes y el grupo control en la fase nocturna del registro, específicamente a partir de las $22 \mathrm{hrs}$ manteniéndose hasta las $2 \mathrm{am}$. Al existir un aumento en el influjo del sistema simpático en las horas de sueño, es comprensible que una de las molestias más frecuentes que reporten estas pacientes sea el sueño de mala calidad; fenómeno que también se ha confirmado en otros estudios con FM y con población masculina y femenina. ${ }^{12,15,33,34}$

Tanto en la medición del índice SDNN como en el de rMSSD, las diferencias significativas se encuentran en el período comprendido entre las $22 \mathrm{hrs}$ y las $8 \mathrm{am}$. Este resultado confirma lo que algunos autores reportan como hiperactividad simpática, ${ }^{35}$ una hiperactividad que resulta altamente disfuncional ya que al alterarse el ciclo de sueño, se facilita la presencia de mayor dolor o cansancio, y con ello, una disminución de las actividades a lo largo del día. Una noche con sueño no reparador entre las personas con FM, es seguida de altos índices de dolor y alteraciones en la atención dirigida al día siguiente. ${ }^{36}$

Las diferencias encontradas entre los grupos en el índice pNN50 en los períodos de medición de las 6 a las 12 hrs, donde el grupo control tiene los valores más altos, reflejan la otra característica de la disautonomía ${ }^{12,13}$ es decir, la disminución del influjo simpático en las pacientes durante un periodo en el que se necesita estar preparados para la acción. Esto lo han reportado la mayoría de las pacientes que acuden a tratamiento dentro del Instituto Nacional de Psi-

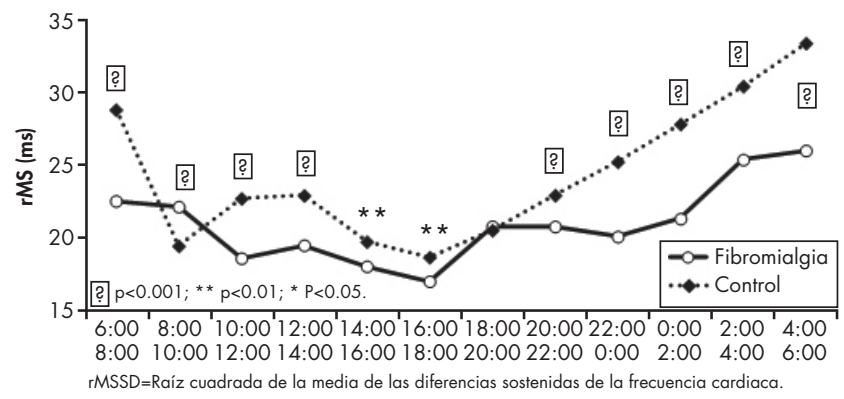

Figura 4. Diferencias en el indicador rMSSD entre pacientes con fibromialgia y controles. 
quiatría Ramón de la Fuente Muñiz, al describir la dificultad que tienen para iniciar sus actividades antes del mediodía.

En nuestra muestra de pacientes con FM, casi la mitad de ellas cursaba con depresión, lo que confirma la alta prevalencia reportada en este padecimiento. ${ }^{21-26}$ Ésta es una de las razones por las que dentro de esta institución psiquiátrica, se tiene desde el 2007 un espacio designado para el tratamiento multidisciplinario de las pacientes con fibromialgia. ${ }^{37}$

Dado que la FM y el uso de antidepresivos alteran la VFC, ${ }^{19,20}$ pensamos que las pacientes que además cursaban con algún trastorno psiquiátrico, podrían haber potencializado aún más una VFC reducida en comparación con personas sanas y con pacientes sólo con FM, sin embargo en nuestro estudio no hubo diferencias significativas para ninguna de las variables entre quienes tenían un diagnóstico psiquiátrico y quienes sólo padecían FM. Aun así, resulta difícil determinar hasta dónde la baja VFC depende de una u otra condición. Aunque se requiere de estudios más controlados y con mayores muestras independientes, la asociación que se ha encontrado entre la disautonomía y la depresión, hace necesario que esta relación sea tomada en cuenta en los tratamientos farmacológicos y no farmacológicos que actualmente se consideran como pilares para su manejo, tales como los antidepresivos y la terapia cognitivo conductual ya que, si bien los medicamentos han demostrado una reducción en algunos síntomas de la depresión, no impactan en la flexibilidad de la VFC. Además, es importante considerar que las pacientes con FM frecuentemente presentan más de un diagnóstico psiquiátrico.

Además de la efectividad que ha mostrado la TCC para

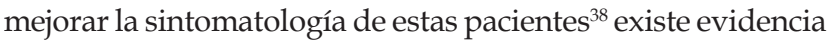
de que la EMT y la RB pueden aumentar la VFC después de controlarse los síntomas depresivos ${ }^{28-30}$ lo que las posiciona como técnicas no invasivas que coadyuvan en el bienestar del paciente al incidir directamente sobre un cambio en la VFC, evitando así la polifarmacia, condición frecuente en estas pacientes por la gran cantidad de síntomas que reportan. ${ }^{39}$

Como lo han sugerido algunos estudios ${ }^{35,40}$ la medición de la VFC puede ser un biomarcador para la FM, sin embargo parece también ser útil en la depresión y otras condiciones de dolor crónico, ${ }^{27}$ ya que como lo refieren algunos autores ${ }^{41,42}$ la FM podría ser parte de los trastornos del espectro afectivo, que comparte, junto con la depresión y otras condiciones médicas y psiquiátricas, elementos patofisiológicos comunes. Es importante que los profesionales de la salud tomen en cuenta esta respuesta en la evaluación y los tratamientos que actualmente ofrecen para estas enfermedades, haciendo uso de las estrategias complementarias para potenciar su efecto.

Es necesario replantearse el impacto del buen funcionamiento del corazón sobre la salud, ya que como lo refieren los hallazgos de la neurocardiología, el corazón parece estar íntimamente involucrado en la coherencia psicofisiológica; ${ }^{43}$ se comunica con el cerebro influyendo en el procesamiento de la información, las percepciones, las emociones y la salud, ${ }^{44}$ enviando mensajes significativos al cerebro que éste entiende y obedece. ${ }^{45}$ Por lo tanto, sólo un modelo holístico que permita una mejor comprensión y manejo de la interacción mentecorazón junto con el conocimiento y empleo de técnicas no invasivas para el análisis y manejo terapéutico de pacientes con enfermedades complejas como la FM, podrá hacer más efectivos los tratamientos, disminuir el riesgo de comorbilidad y mejorar la calidad de vida de estas pacientes.

\section{Limitaciones y sugerencias}

Es importante considerar en futuros estudios la comparación de la VFC en pacientes con depresión vs. pacientes con FM solamente vs. pacientes con FM más depresión, aplicando una clinimetría que incluya el grado de severidad tanto de los trastornos psiquiátricos, como de la FM. Se sugiere ampliar la muestra y controlar el tipo de antidepresivo y dosis para tener una mayor precisión en los resultados obtenidos. Así también, se sugiere contar con datos que den cuenta de la forma cómo las pacientes experimentan los síntomas para confirmar y/o aclarar los datos obtenidos con los instrumentos empleados. Por ejemplo, llevar una hoja de registro donde se consideren variables como la hora de acostarse, la hora del despertar, la presencia de actividad física, y la alimentación, así como la presencia de eventos estresantes y la forma como se afrontaron.

\section{Financiamiento}

Ninguno.

\section{Conflicto de intereses}

Los autores declararon no tener conflicto de intereses.

\section{REFERENCIAS}

1. Goldenberg D. Fibromialgia. Una guía completa para comprender y aliviar el dolor. Barcelona, España: Paidós; 2003.

2. Singh KD, Stauth C. Curar el dolor. México: Urano; 2001.

3. Bennett RM. Clinical manifestations and diagnosis of fibromyalgia. Rheum Dis Clin North Am 2009;35:215.

4. Podolecki T, Podolecki A, Hrycek A. Fibromyalgia: pathogenic, diagnostic and therapeutic concerns. Polskie Archiwum Medycyny Wewnetrznej 2009;119(3):157-160.

5. Fietta P. Fibromyalgia: state of the art. Minerva Medica 2004;95(1):35-52.

6. Staud R, Spaeth M. Psychophysical and neurochemical abnormalities of pain processing in fibromyalgia. CNS Spectr 2008;13(3)(suppl 5):12-17.

7. Servant D, Logier R, Mouster Y, Goudemand M. Heart rate variability. Applications in psychiatry. Encephale 2009;35(5):423-428.

8. Stein PK, Kleiger RE. Insights from the study of heart rate variability. Annual Reviews Medicine 1999;50:249-261.

9. Tarvainen MP, Georgiadis SD, Ranta-aho PO, Karjalainen PA. Timevarying analysis of heart rate variability signals with Kalman smoother algorithm. Physiological Measurements 2006;27(3):225-239.

10. Andreassi J. Psychophysiology. Human behavior \& physiological response. New Jersey: Erlbaum; 2000. 
11. Martínez-Lavín M, Hermosillo AG, Mendoza C, Ortiz R et al. Orthostatic sympathetic derangement in subject with fibromyalgia. J Rheumatology 1997;24:714-718.

12. Martínez-Lavín M, Hermosillo AG, Rosas M, Soto ME. Circadian studies of autonomic nervous balance in patients with fibromyalgia. A heart rate variability analysis. Arthritis Rheumatism 1998;42:1966-1971.

13. Martínez-Lavín M, Hermosillo, AG. Autonomic nervous system dysfunction may explain the multisystem features of fibromyalgia. Seminars Arthritis Rheumatism 2000;29:197-199.

14. Cohen H, Neumann L, Shore M, Amir M et al. Autonomic dysfunction in patients with fibromyalgia; application of power spectral analysis of heart rate variability. Semin Arthritis Rheum 2000;29:217-227.

15. Raj RR, Brouillard D, Simpsom CS, Hopman WM et al. Dysautonomia among patients with fibromyalgia: A noninasive assessment. J Rheumatology 2000;27:2660-2665.

16. Udupa K, Sathyaprabha TN, Thirthalli J, Kishore KR et al. Modulation of cardiac autonomic functions in patients with major depression treated with repetitive transcranial magnetic stimulation. J Affect Disord 2007;104:231-236.

17. Van der Kooy KG, Van Flout HP, Van Marwijk HW, de Haan M et al. Differences in heart rate variability between depressed and nondepressed elderly. Int J Geriatr Psychiatry 2006;21:147.

18. Licht CMM, de Geus EJC, Zitman FG, Hoogendijk WIG et al. Association between major depressive disorder and heart rate variability in the Netherlands study of depression and anxiety (NESDA). Arch Gen Psychiatry 2008;65:1358-1367.

19. Kemp AH, Quintana DS, Gray MA, Felmingham KL et al. Impact of depression and antidepressant treatment on heart rate variability: a review and meta-analysis. Biol Psychiatry 2010;67(11):1067-1074.

20. Glassman AH, Bigger JT, Gaffney M, Van Zyl LT. Heart rate variability in acute coronary syndrome patients with major depression: influence of sertraline and mood improvement. Arch Gen Psychiatry 2007;64(9):1025-1031.

21. Ahles TA, Khan SA, Yunus MB, Spiegel DA et al. Psychiatric status of patients with primary fibromyalgia, patients with rheumatoid arthritis, and subjects without pain: a blind comparison of DSM-III diagnoses. Am J Psychiatry 1991;148(12):1721-1726.

22. Epstein SA, Kay G, Clauw D, Heaton R et al. Psychiatric disorders in patients with fibromyalgia. A multicenter investigation. Psychosomatics 1999;40(1):57-63.

23. Hudson JI, Arnold LM, Keck PE Jr, Auchenbach MB et al. Family study of fibromyalgia and affective spectrum disorder. Biol Psychiatry 2004;56(11):884-891.

24. Arnold LM, Hudson JI, Keck PE, Auchenbach MB et al. Comorbidity of fibromyalgia and psychiatric disorders. J Clin Psychiatry 2006;67(8):1219-1225.

25. McBeth J, Silman AJ. The role of psychiatric disorders in fibromyalgia. Curr Rheumatol Rep 2001;3(2):157-164.

26. Martinez JE, Ferraz MB, Fontana AM, Atra E. Psychological aspects of Brazilian women with fibromyalgia. J Psychosom Res 1995;39(2):167-174.

27. Mostoufi S, Afar N, Ahumada M, Reis V et al. Health and distress predictors of heart rate variability in Fibromyalgia and other forms of chronic pain. J Psychosomatic Researh 2012;72:39-44.
28. Lehrer PM, Vaschillo E, Vaschillo B. Resonant frequency biofeedback training to increase cardiac variability: Rationale and manual for training. Applied psychophysiology Biofeedback 2012;25(3):177-191.

29. Udupa K, Sathyaprabha TN, Thirthalli I, Kishore KR et al. Alteration of cardiac autonomic functions in patients with major depression: A study using heart rate variability measures. J Affect Disord 2007;100:137-141.

30. Nahshoni E, Aizenberg D, Sigler M, Zalsman G et al. Heart rate variability in elderly patients before and after electroconvulsive therapy. Am J Geriatr Psychiatry 2001;9:255-260.

31. Sheehan DV, Lecrubier Y, Sheehan KH, Amorim Pet al. The Mini-International Neuropsychiatric Interview (M.I.N.I.): the development and validation of a structured diagnostic psychiatric interview for DSM-IV and ICD-10. J Clinical Psychiatry 1998;59(Suppl 20):34-57.

32. Wolfe F, Smythe HA, Yunus MB, Bennett RM et al. American College of Rheumatology 1990 criteria for the classification of fibromyalgia. Arthritis Rheumatism 1990;33(2):160-172.

33. Martínez-Lavín M. A novel holistic explanation for the fibromyalgia enigma. Autonomic nervous system dysfunction. Fibromyalgia Frontiers 2002;10(1):3-12.

34. Cohen H, Neumann L, Alhosshle A. Abnormal sympathovagal balance in men with fibromyalgia. J Rheumatology 2001;28:581-589.

35. Lerma C, Martínez A, Ruiz N, Vargas A et al. Nocturnal heart rate variability parameters as potential fibromyalgia biomarker. Correlation with symptoms severity. Arthritis Research Therapy 2011;13:R185.

36. Moldofsky $\mathrm{H}$. The significance, assessment and management of nonrestorative sleep in fibromyalgia syndrome. CNS Spectr 2008;13(3)(Suppl 5):22-26.

37. Barrera MI. Programa de intervención multimodal en pacientes con fibromialgia. Tesis de doctorado, Facultad de Psicología, UNAM, México; 2007.

38. Goldenberg D. Manejo clínico de la fibromialgia. México: Intersistemas, S.A: de C.V.; 2009.

39. Martínez-Lavín M. Fibromialgia. Cuando el dolor se convierte en enfermedad. España: Santillana Ediciones Generales; 2006.

40. Staud R. Heart rate variability as a biomarker of fibromyalgia syndrome. Fut Rheumatolog 2008;3(5):475-483.

41. Hudson JI, Pope HG Jr. Fibromyalgia and psychopathology: is fibromyalgia a form of "affective spectrum disorder"? J Rheumatol 1989;(Suppl 19):15-22.

42. Hudson JI, Arnold LM, Keck PE Jr, Auchenbach MB et al. Family study of fibromyalgia and affective spectrum disorder. Biol Psychiatry 2004;56(11):884-891.

43. McCraty R. Heart Rhythm coherence. An emerging area of biofeedback. HeartMath Research Center, Institute HeartMath 2002;30(1):23-25.

44. Armour JA. Cardiac neuronal hierarchy in health and disease. Am J Physiol Regul Integr Comp Physiol 2004;287(2):R262-271.

45. Lacey BC, Lacey JI. Two-way communication between the heart and the brain. Significance of time within the cardiac cycle. Am Psychol 1978;33(2):99-113. 